\title{
Consideration of input thermal wave frequency for minimizing inspection time of active thermography
}

\author{
by M. Ishikawa*, S. Ishihara*, H. Nishino*, M. Koyama**, H. Kasano***, \\ H. Hatta****, and S. Utsunomiya ${ }^{\star \star \star \star}$ \\ *Tokushima University, 2-1 Minamijousanjima-cho, Tokushima, 770-8506, Japan, m.ishikawa@tokushima-u.ac.jp \\ ${ }^{* *}$ Meisei University, 2-1-1 Hodokubo, Hino, Tokyo, 191-8506, Japan \\ *** Nihon University, Tokusada, Tamura, Koriyama, Fukushima,963-8642, Japan

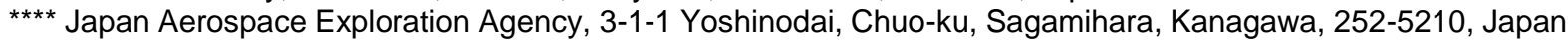

\begin{abstract}
To shorten the inspection time of active thermographic non-destructive testing is desired to enhance its inspection efficiency. The required inspection time should strongly depend on the frequency of the input thermal wave, as it influences the heat propagation speed. In this study, the relationship between the frequency component of the input thermal wave and the inspection time is discussed through fundamental experiments and numerical calculations. The results showed that the input frequency component was varied by changing heating method, and the inspection time was shortened when using thermal wave with higher frequency components that have higher heat propagation speed.
\end{abstract}

\section{Introduction}

Active thermographic inspection is a convenient non-destructive testing method; it can be used to inspect a relatively large area in a single test without physical contact with the test object. However, the active thermographic inspection presents a practical limitation: a relatively long inspection time is required when inspecting low-thermal-diffusivity materials (such as polymer materials or concrete structures) because heat propagation speed in such materials is slow. This limitation leads to a degraded efficiency of the inspection process, thus shortening the inspection time is often desired. The aim of this study is to minimize the inspection time of active thermography by considering thermal wave theory and the frequency of the input thermal wave. According to the fundamental heat propagation theory, the heat propagation speed is proportional to the frequency of the thermal wave. When a periodic temperature variation is generated on the surface of an object, the heat propagation speed in the object $(v)$ is obtained from the one-dimensional thermal wave theory as $[1,2]$

$$
v=\sqrt{\pi \alpha f}
$$

where $\alpha$ and $f$ are the thermal diffusivity of the object and frequency of the periodic temperature change, respectively. This equation indicates that $v$ increases with increasing $f$. Therefore, by using thermal waves with high frequency components, the inspection time is expected to shorten because the input heat propagates to the subsurface defects in a shorter time. However, thermal waves are also known to get attenuated during propagation, and the attenuation ratio drastically increases with $f$.

In this study, we investigated the relationship between the frequency components of thermal waves input to a test object and the time required for detecting subsurface defects through experiments and numerical simulations. Our previous studies found that the required minimum inspection time is affected by the frequency of the periodic heating $[3,4]$. In the present study, we performed a fundamental study to further discuss the relationship between the frequency components of the input thermal waves and the required inspection time for active thermography. Polymethylmethacrylate (PMMA) specimens with an artificial defect were prepared and heated using periodic heating with different frequencies. By varying the heating method, the frequency components of the input thermal waves also varied. Thus, the actual input frequency components were investigated in relation to the time required to detect the defect.

\section{Experiments}

\subsection{Experimental setup}

A picture and a schematic of a PMMA specimen are shown in figure 1 . The specimen dimensions were $150 \times$ $150 \times 20 \mathrm{~mm}$, and it has a $50 \mathrm{~mm}$ wide flat-bottomed groove acting as an artificial defect. Two specimens were prepared, 
and the defect depths from the observed surface were 3 and $5 \mathrm{~mm}$, respectively. The specimens were heated using a 500 W halogen lamp, and the surface temperature during heating was monitored by an infrared camera (A315, FLIR Systems, Inc.). The output power of the lamp was controlled by a function generator (AFG3102, Tektronix) to sine-shaped periodic heating. The heating frequency varied as $0.01,0.03,0.05$, and $0.1 \mathrm{~Hz}$. The minimum time required to detect the artificial defect (denoted as $t_{\mathrm{min}}$ ) was then investigated under each heating frequency condition. Note that the $t_{\text {min }}$ value was determined using phase data obtained by applying Fourier transform to the observed temperature data $[5,6,7,8](\mathrm{a}$ faint temperature change occurring in the earlier stage of the observation process can be detected more easily in the phase images than in the thermal images). In figure 2, an example of observed temperature-time data is shown. Fourier transform was applied to the temperature data for an arbitrary time from the initial time, and the relationship between the signal-tonoise ratio $(S / N)$ in the phase image and the time duration of the Fourier transformed temperature data was obtained. In the calculation of the $S / N, S$ was determined as the phase contrast between defective and non-defective area, and $N$ was the standard deviation of the phase values in non-defective area. The $t_{\min }$ was then determined as the time when $S / N$ reached a value of 2

(a)

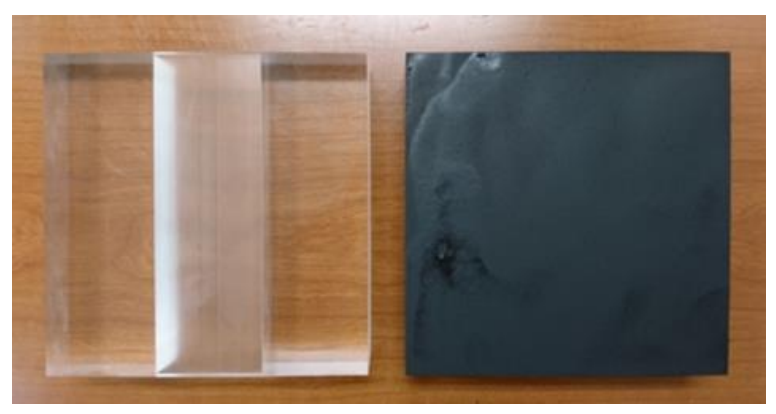

(b)

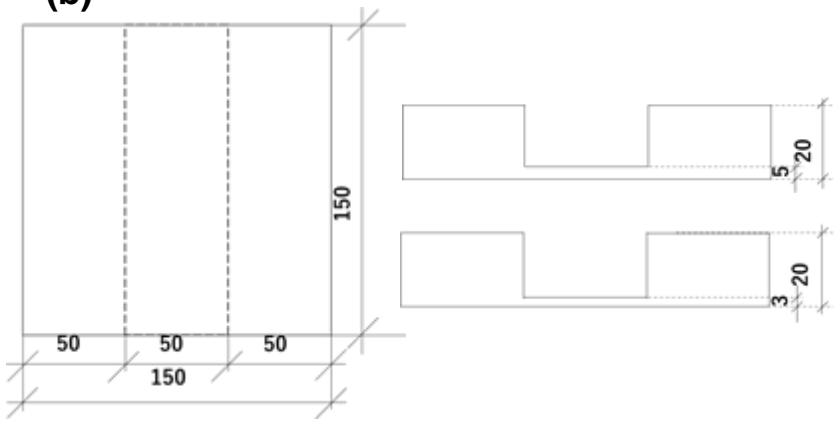

Fig. 1. (a) picture and (b) schematic illustration of the PMMA specimen.

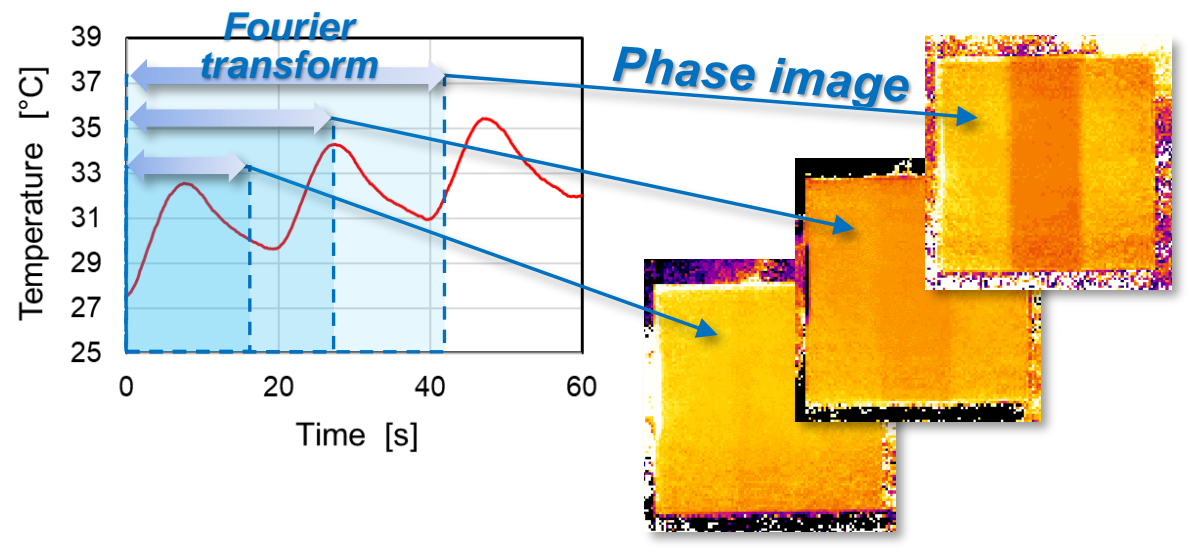

Fig. 2. Example of observed temperature-time data and phase images obtained by applying Fourier transform to the temperature data for an arbitrary time.

\subsection{Results}

The phase images obtained from the specimens using $0.05 \mathrm{~Hz}$ heating are shown in figure 3 . The artificial defect is observed as an area with different phase values, and observed more clearly as time elapses (figure 3(b) and 3(e) are the phase image when the $S / N=2$ ). Figure 4 shows the relationship between the $t_{\min }$ (the time when the $S / N=2$ in the phase images) and the heating frequency for both specimens. In the same figure, the theoretical estimations of $t_{\text {min }}$ values for detecting 3- and 5-mm-depth defects are presented as solid lines. These theoretical values were calculated using Eq. (1) as a round-trip propagation time between the surface and subsurface defects. These results show that, although $t_{\mathrm{min}}$ decreases with increasing the heating frequency from 0.01 to $0.03 \mathrm{~Hz}$ along the theoretical estimation, further reduction is 
not observed when the heating frequency is 0.05 and $0.1 \mathrm{~Hz}$. A likely reason for this is due to the attenuation of heat during propagation. Thermal waves with higher frequencies are more drastically attenuated; hence, the input thermal wave with a high frequency component could not return to the observation surface, and thermal waves with a lower frequency which were input simultaneously contributed to defect detection instead. These results suggest that a detailed investigation of the frequency components of the input and returned thermal waves is important.

(a)

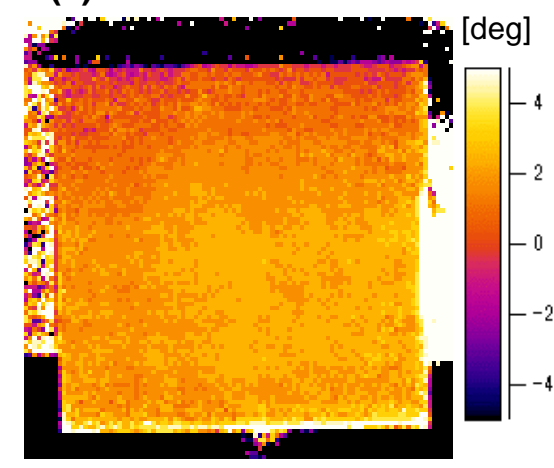

(d)

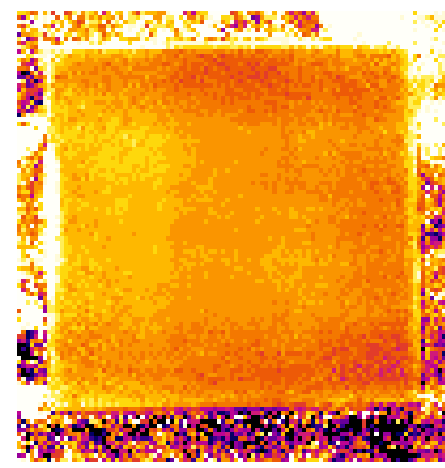

(b)

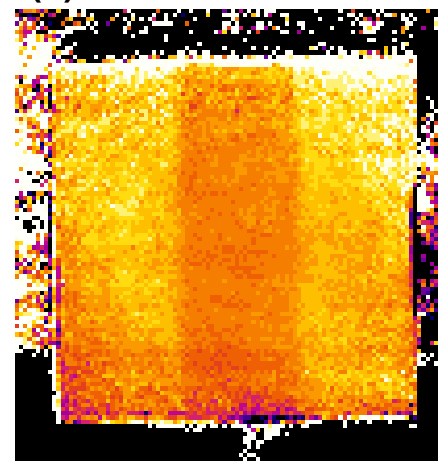

(e) (c)

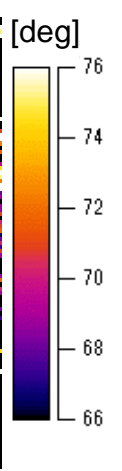

[deg]

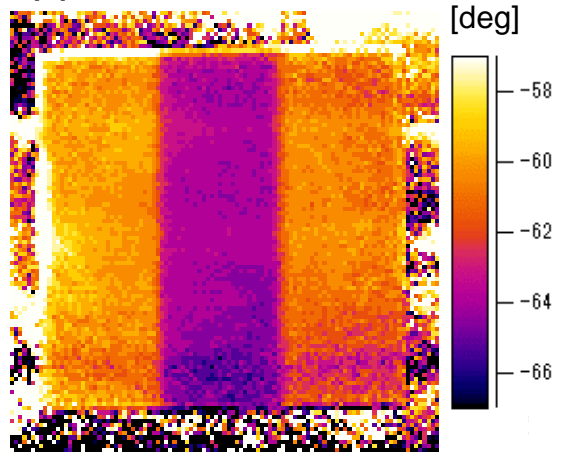

(f)

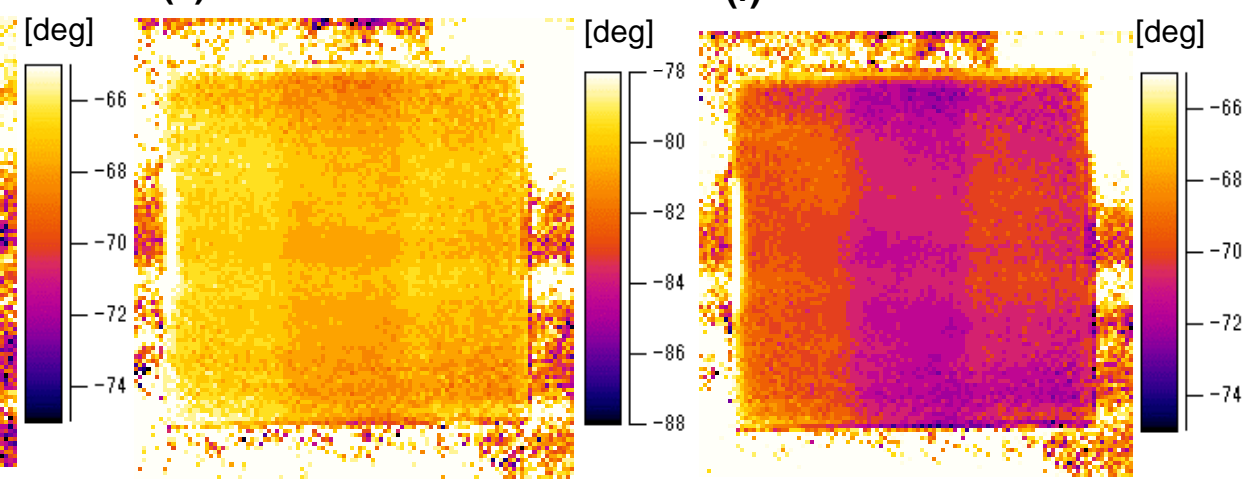

Fig. 3. Phase images for the specimen with (a)-(c) a 3-mm-depth defect and (d)-(f) a 5-mm-depth defect obtained using $0.05 \mathrm{~Hz}$ heating from temperature-time data for (a) $24.8 \mathrm{~s}$, (b) $34.8 \mathrm{~s}(\mathrm{~S} / \mathrm{N}=2)$, (c) $44.8 \mathrm{~s}$, (d) $67.2 \mathrm{~s},(e) 77.2 \mathrm{~s}(\mathrm{~S} / \mathrm{N}=2)$, and (f) $87.2 \mathrm{~s}$.

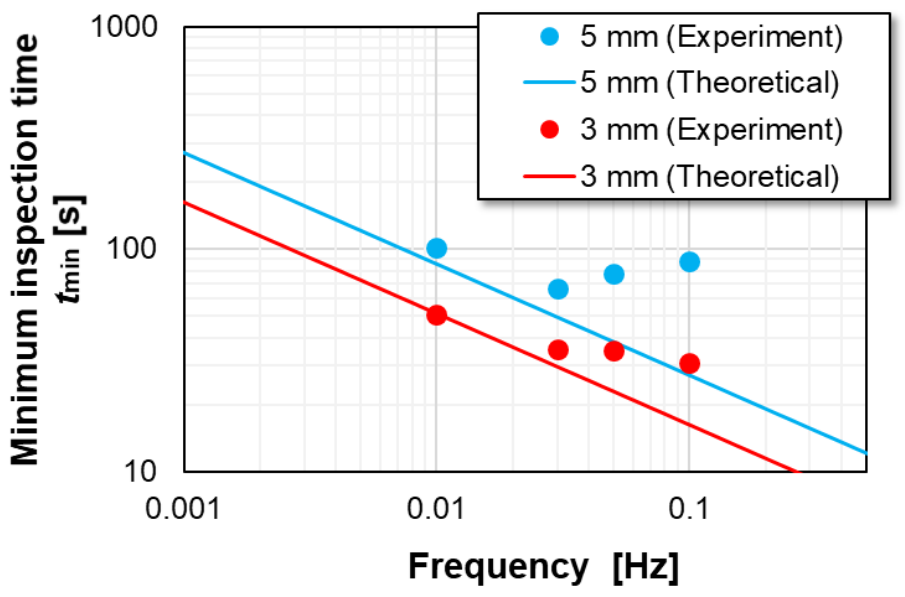

Fig. 4. Relationship between the experimentally obtained minimum inspection time $t_{\min }$ and heating frequency (dots) and theoretically estimated minimum inspection time as a function of thermal wave frequency (solid lines). 


\section{Investigation of frequency components of the input thermal wave}

When an object is heated periodically, thermal waves with various frequency components should be input, not only the component corresponding to the heating frequency. Therefore, the frequency content of the input thermal wave was investigated through simple numerical calculations.

In order to simulates the experiments, a PMMA model (density, specific heat and thermal conductivity are 1190 $\mathrm{kg} / \mathrm{m}^{3}, 1470 \mathrm{~J} / \mathrm{kgK}$ and $0.19 \mathrm{~W} / \mathrm{mK}$, respectively) was prepared, and periodic heat load was applied to the model surface.
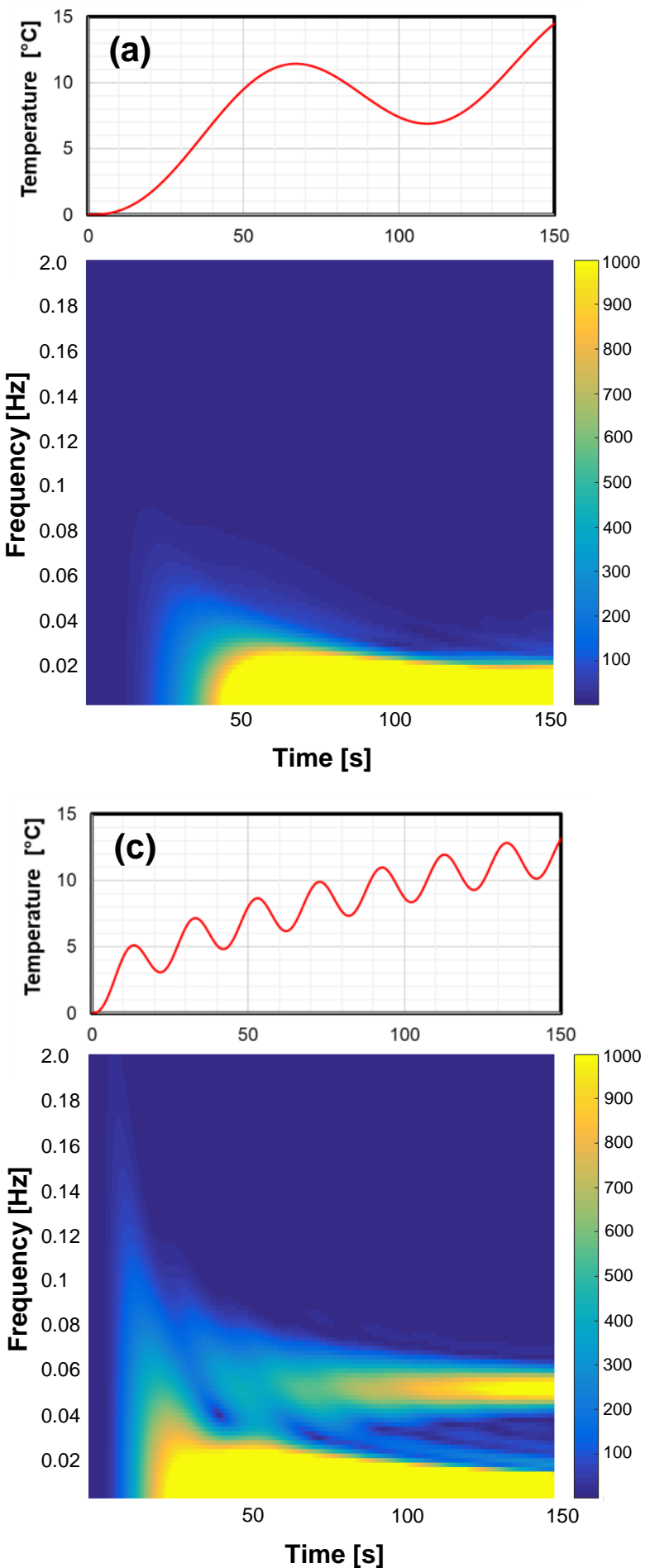
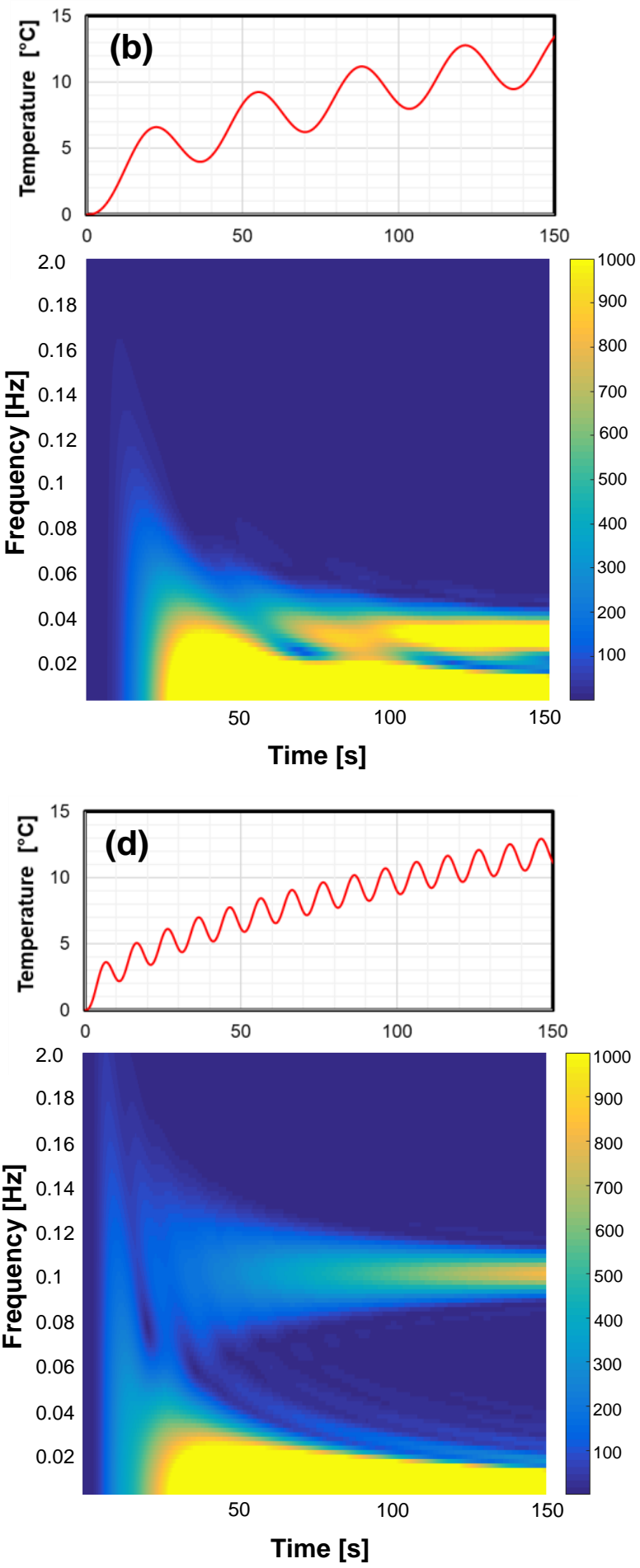

Fig. 5. Numerically calculated temperature-time data in the heated surface and spectral amplitude as a function of time obtained when heating frequency is (a) $0.01 \mathrm{~Hz}$, (b) $0.03 \mathrm{~Hz}$, (c) $0.05 \mathrm{~Hz}$, and (d) $0.1 \mathrm{~Hz}$. 

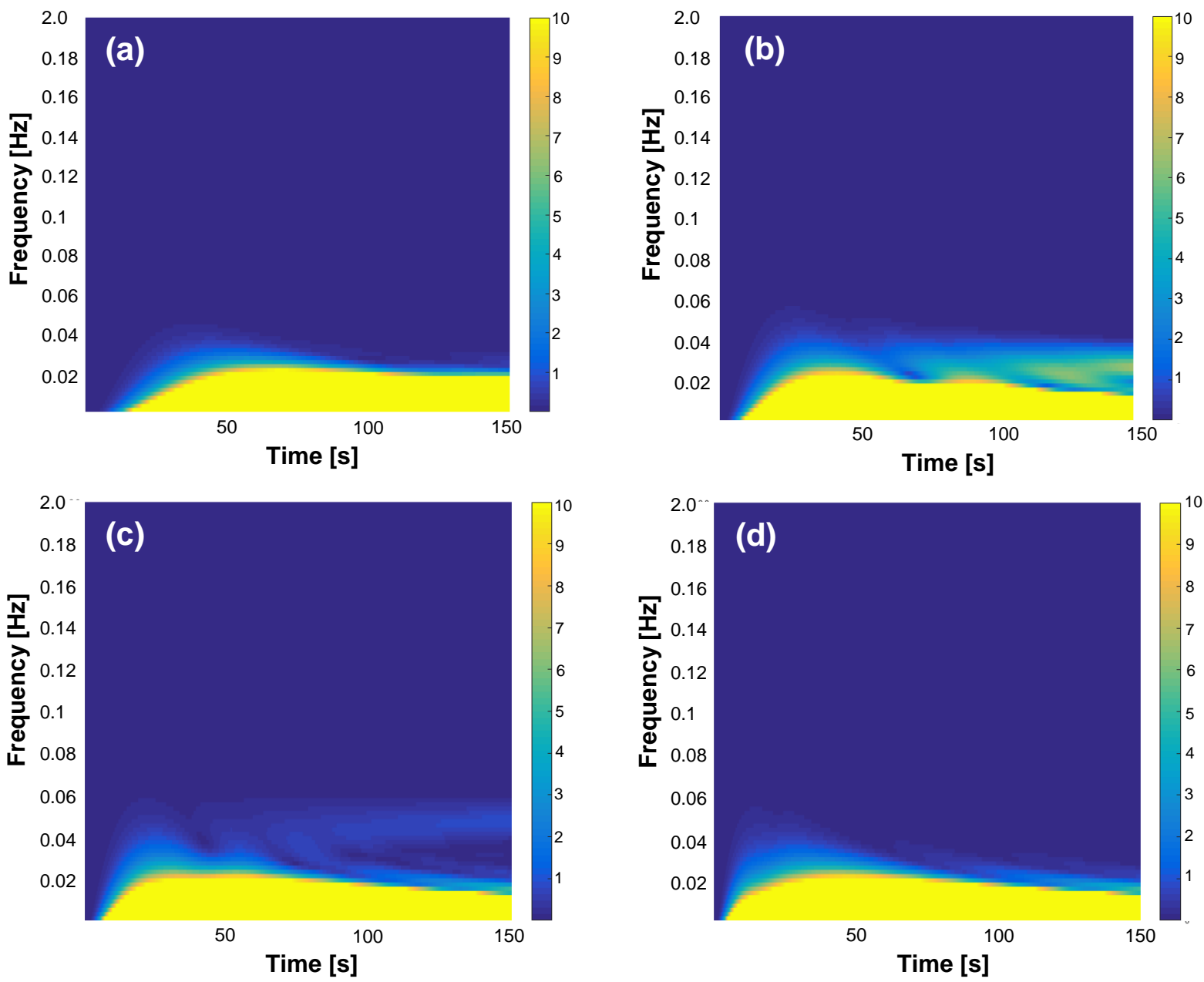

Fig. 6. Calculated spectral amplitude in consideration of the attenuation caused by round-trip propagation to a 3-mm-depth defect obtained when heating frequency is (a) $0.01 \mathrm{~Hz}$, (b) $0.03 \mathrm{~Hz}$, (c) $0.05 \mathrm{~Hz}$, and (d) $0.1 \mathrm{~Hz}$.

The thickness of the model was large enough to be considered as a semi-infinite solid, and the heat load was applied uniformly to the surface. Thus, the model substantially simulates the one-dimensional heat propagation. The frequency of the periodic heat load was varied as the same frequencies as those used in the experiments $(0.01,0.03,0.05, a n d 0.1 \mathrm{~Hz})$. Under each heating frequency condition, the surface temperature during heating was obtained, and the spectral amplitude of the obtained temperature-time data was calculated by applying Fourier transform in the same manner as the experiments (figure 2).

Figure 5 shows the calculated temperature-time data and spectral amplitude as a function of the elapsed time from heating start (the vertical and horizontal axes indicate the frequency and elapsed time, respectively, and the color distribution shows the spectral amplitude). By comparing the spectral amplitude for each heating condition, it is found that thermal waves with larger amplitude and higher frequency are input in shorter time when the heating frequency is higher. This means the thermal waves with higher propagation speed is input in shorter time by increasing the heating frequency, and this should be the reason for the reduction of $t_{\min }$ in the experiments. Figure 6 shows the spectral amplitude of the thermal waves after propagating the round-trip distance between the surface and 3-mm-depth defect calculated by considering the attenuation (the attenuated amplitude was obtained by multiplying $\exp (-z \sqrt{\pi f / \alpha})$, where $z$ denotes the propagation distance). It is found from figure 6 that the spectral amplitude significantly decreases due to the attenuation, and the thermal waves with the frequency higher than $0.05 \mathrm{~Hz}$ is hardly detected in all heating conditions. This means that thermal waves with such higher frequency cannot return from the defect, and that further increasing the input frequency is not effective to shorten the inspection time. This should be the reason for not decreasing $t_{\min }$ when the heating frequency was 0.05 and $0.1 \mathrm{~Hz}$ in the experiments. Furthermore, this result implies the $t_{\min }$ values obtained when using $0.05 \mathrm{~Hz}$ heating $(34.8 \mathrm{~s})$ is nearly the minimum time that can be shortened by changing the input thermal wave frequency. The attenuation caused by the round-trip propagation to the $5-\mathrm{mm}$-depth defect is also examined, and the result showed thermal waves with the frequency higher than approximately $0.02 \mathrm{~Hz}$ is difficult to detect. Thus, for the detection of the 5-mm-depth defect, the minimum inspection time should nearly be the $t_{\min }$ obtained when the heating frequency is $0.03 \mathrm{~Hz}(66.1 \mathrm{~s})$. 


\section{Conclusion}

In order to shorten the inspection time of active thermography method, using thermal waves with higher frequency components was focused on, and its effect was examined with the investigation of the frequency components of the input and returned thermal waves. Experimental results showed that, although the inspection time was reduced with increasing the heating frequency, the time reduction effect was small when the heating frequency was high. This should be because, although the thermal waves with higher frequency components are input in shorter time when the heating frequency is higher, the higher frequency components are attenuated more significantly and contribute little to the defect detection. This shows a limitation of reducing inspection time from the view point of this study. A possible way to inspect in a further short time is to input larger heat energy enough to detect the returned thermal waves with high frequency components. However, there is also a limitation regarding the heat resistant temperature of the inspection object; it should be a practical factor to determine the possible minimum inspection time.

\section{Acknowledgements}

This work was supported by Japan Society for the Promotion of Science (JSPS) KAKENHI Grant Numbers 17K01296.

\section{REFERENCES}

[1] Almond D.P., Patel P., "Photothermal Science and Techniques", Chapman and Hall, London (1996).

[2] Maldague X., "Theory and practice of infrared technology for nondestructive testing", John Wiley \& Sons, New York (2001).

[3] Ishikawa M., Koyama M., Kasano H., Fukui R., Hatta H., Utsunomiya S., Nishino H. " Reducing inspection time of active thermographic non-destructive testing based on thermal wave theory", Proceedings of 3rd Quantitative InfraRed Thermography Asia conference, paper QIRT-2019-009 Tokyo (Japan), 2019.

[4] Ishikawa M., Ishihara S., Nishino H., Koyama M., Kasano H., Fukui R., Hatta H., Utsunomiya S. " Relationship between Inspection Time and Frequency Components of Input and Reflected Thermal Waves in Active Thermographic Non-Destructive Inspection", Proceedings of 15th International Workshop on Advanced Infrared Technology and Applications (AITA 2019), Florence (Italy), 2019.

[5] Maldague X., Marinetti S., Pulse phase infrared thermography, J. appl. Phys. - Vol. 79, no 5, pp. 2694-2698, 1996.

[6] Marinetti, S., Plotnikov, Y.A., Winfree, W.P., Braggiotti, A., Pulse phase thermography for defect detection and visualization. Nondestructive Evaluation of Aging Aircraft, Airports, and Aerospace Hardware III (Vol. 3586, pp. 230-238), International Society for Optics and Photonics, 1999.

[7] Weritz F., Arndt R., Röllig M., Maierhofer C., Wiggenhauser H., Investigation of concrete structures with pulse phase thermography, Materials and Structures. - Vol. 38, no 9, pp. 843-849, 2005.

[8] Ishikawa M., Hatta H., Habuka Y., Fukui R., Utsunomiya S., Detecting deeper defects using pulse phase thermography. Infrared Phys. Technol. - Vol. 57, pp. 42-49, 2013. 